\title{
MODELLING OF THE GROWTH AND DETACHMENT OF A VAPOUR BUBBLE AND THE EFFECT OF AN ELECTRIC FIELD IN THE NUCLEATE BOILING REGIME
}

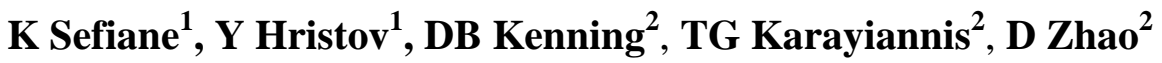 \\ ${ }^{1}$ University of Edinburgh, Edinburgh, UK \\ ${ }^{2}$ Brunel University, London, UK
}

\begin{abstract}
A comprehensive model predicting the deformation, growth and detachment of a vapour bubble in the nucleate boiling regime with an applied electric field is described in this paper. The model takes into account the full electrohydrodynamics of the phenomenon including the influence of local temperature on the generation of free charges in the liquid. Solution of the model by the level set method has been successfully implemented with a commercial CFD code. Aspects of the code and the graphical software requiring further development are noted.

Sample results are presented to demonstrate the effect of the electric field on the growth and detachment of the bubble, for a bubble initially protruding through a thermal boundary layer on a horizontal wall. The bubble is elongated under the influence of electrical forces, the effect being more pronounced for stronger electrical fields. The electric field is found to promote earlier detachment of the bubble at a smaller volume, thus increasing the bubble frequency. The wall heat flux during the process of detachment is not much affected by the electric field.
\end{abstract}

\section{Nomenclature}

$c_{P}$ - specific heat at constant pressure, $J / \mathrm{Cg}^{-}$

$T$ - temperature, $K$

$E$ - electric field strength, $V / m$

g - gravitational vector, $m / s^{2}$

$H$ - Heaviside step function

$h$ - grid spacing

$h_{f g}$ - latent heat of evaporation, $J / \mathrm{kg}$

$\Delta T_{\text {sat }}=T_{w}-T_{\text {sat }}$ - wall superheat, $K$

$J$ - electric current density, $A / m^{2}$

$k$ - thermal conductivity, $W / \mathrm{n} K$

m - mass flux vector, $\mathrm{kg} / \mathrm{C}^{2} \mathrm{~s}$

$\dot{m}_{\text {mic }}$ - evaporation rate from the microlayer,

$\mathrm{kg} / \mathrm{s}$

$p$ - pressure, $N / m^{2}$

$q$ - wall heat flux, $W / m^{2}$

$r$ - coordination, $m$

$R$ - radius of computational domain, $m$

$R_{o}$ - radius of dry region beneath a bubble, $m$

$R_{1}$ - radial location of the interface at $y=h / 2$,

$m$

$t$ - time, $s$

$\mathbf{u}$-velocity vector $\boldsymbol{Q}, v$

$u$-r-directional velocity, $\mathrm{m} / \mathrm{s}$

$v$ - y-directional velocity, $\mathrm{m} / \mathrm{s}$

$V$ - bubble volume, $\mathrm{m}^{3}$

$V_{c}$ - control volume, $\mathrm{m}^{3}$

$y$ - vertical coordinate, $m$

$Y$ - height of computational domain, $m$

\section{Greek symbols}

$\alpha$ - thermal diffusivity of liquid, $\mathrm{m}^{2} / \mathrm{s}$

$\alpha_{K}$ - coefficient of thermal increase of electric conductivity of R123, $1 / K$

$\beta_{T}$ - coefficient of thermal expansion, $1 / K$

$\delta$ - thickness, $m$

$\delta_{\varepsilon} \phi$-delta function

$\delta_{T}$ - thermal boundary layer thickness, $m$

$\varepsilon$ - electric permittivity, $F / m$ 
$\kappa$ - interface curvature, $1 / m$

$\mu$ - dynamic viscosity, N.s $/ \mathrm{m}^{2}$

$\gamma$ - kinematic viscosity, N.s. $\mathrm{m} / \mathrm{kg}$

$\rho$ - mass density, $\mathrm{kg} / \mathrm{m}^{3}$

$\rho_{e}$ - electric charge density, $C / \mathrm{m}^{3}$

$\sigma$ - surface tension, $N / m$

$\sigma_{\mathrm{e}}$ - electric conductivity, $S$

$\phi$ - level set function

$\Phi$ - electric potential, $V$ $\varphi$ - apparent contact angle, deg

$\psi$-initial bubble shape profile

\section{Subscripts}

int -interface

$l$ - liquid

sat - saturation

$v$ - vapour

$w$-wall interface

\section{Introduction}

Nucleate boiling is a very efficient mode of heat transfer involving liquid evaporation associated with bubble nucleation, growth and detachment. Due to its importance in many engineering applications it has been subject to extensive investigations over the years. Achieving higher heat transfer rates through various enhancement techniques can result in considerable decrease in the size of heat exchangers as well as improvement of their performance, which leads to substantial energy savings. Enhancement techniques can be categorised into two groups: passive and active. Whilst passive techniques may require fluid additives or special surface geometries, active techniques use external forces such as electric field, acoustic or surface vibrations. Many experimental works have shown the great potential of Electrohydrodynamics (EHD) in enhancing boiling heat transfer- Karayiannis and Allen (1991), Ogata and Yabe (1993), Karayiannis (1998) and etc.. Reviews of these works have been undertaken by Allen and Karayiannis (1995) and recently by Laohalertdecha et al. (2005). Compared to experimental works on the subject, available theoretical investigations are rather scarce and limited. One possible reason is that the mechanism of boiling heat transfer itself is still poorly understood. Adding the effect of an electric field makes the modelling even more complicated. The augmentation of boiling heat transfer by utilizing the EHD effects has been recently modelled for different boiling regimes: in nucleate boiling, Zaghdoudi and Lallemand (2002), in saturated boiling near critical heat flux (CHF), Di Marco and Grassi (1999) and in film boiling, Verplaetsen and Berghmans (1999). Most of these models adopt assumptions and use empirical correlations, which makes them less general. On the other hand visual observations during boiling showed that applying electric field affects single bubble behaviour. It is thus reasonable to suggest that EHD enhances nucleate boiling heat transfer through the effect on bubble's growth and detachment. Another very important observation is that the EHD enhancement effects are much more remarkable with increasing the applied voltage. It is also shown that higher magnitudes of electric field strength cause more significant deformation of the bubble.

Recently Karayiannis and $\mathrm{Xu}$ (1998) described the effect of electric forces on a single bubble during nucleate boiling regime. They used R123 electric properties and applied voltage from 0 to 20 $\mathrm{kV}$ for the simulations. The most important idea of the work is that the bubble can be included as part of the domain of interest and thus the definition of a boundary condition at the bubble interface becomes unnecessary. Their results did not consider the deformation of the bubble, but instead they investigated spherical bubble and an array of bubbles. Oh and Kwak (2000) presented theoretical and experimental results for bubble behaviour during boiling under DC electric field condition. R11, R113 and FC72 were used for the experiments and the numerical calculations in up to $20 \mathrm{kV}$ and electrode gap form 5 to $8 \mathrm{~mm}$. Their results consider only the electric force distribution around the bubble and its dependence on the electrode configuration, but not the deformations of the bubble. Zaghdoudi and Lallemand (2000) presented a study of the behaviour of a bubble in uniform electric field with up to $20 \mathrm{kV} / \mathrm{cm}$ strength. They obtained the electroconvective movements around and within a conducting bubble and its deformation. Only small deviations from an initially 
spherical bubble shape and stationary bubble could be mentioned as a limitation of that model. Herman et al. (2004) reported experimental and theoretical results on bubble deformation and detachment under the influence of electric field in terrestrial and microgravity conditions. They used an experimental set-up where air is pumped through an orifice in the liquid to emulate bubble volume growth during boiling. In this work the authors modelled the above experiment with electric field strengths up to $10 \mathrm{kV} / \mathrm{cm}$. In the model they do not consider changes of the electric properties of the liquid with temperature which has been shown by Turnbull (1968) to be very important. Zhang et al. (2003) studied numerically EHD effects on an isolated bubble attached on a horizontal heating surface in nucleate boiling of R134a. Mathematical and numerical models for the heat and fluid flows surrounding the spherical bubble were proposed. The bubble dynamics and the flow structure and temperature field in the nucleate boiling region were analysed. The results showed that EHD can strongly enhance the heat transfer rate of nucleate boiling by destabilising the flow structure in the vicinity of the heating surface. No deformation of the bubble was considered in the presented work.

The modelling of the effect of an electric field on the growth and detachment of a single bubble during nucleate boiling heat transfer is far from being complete. Most of the models reviewed above focus on limited aspects of the complete cycle of nucleation, growth and detachment of a deformed bubble in the presence of an electric field. There have been CFD simulations of the bubble cycle without an electric field, e.g. by Son et al. (1999) employing the level-set method. The model for the growth and detachment of a bubble in the presence of an electric field described in the present paper is based on the level set formulation (Son et al. (1999)) combined with the EHD model proposed by Karayiannis and Xu (1998).

Following the strategy of Son et al. (1999), the model combines sub-models for the micro-region of the thin liquid film under a bubble, strongly influenced by surface forces, and the macro-region surrounding the bubble, in which conventional thermofluid equations apply. Progress in developing a model for the micro-region in the presence of an electric field has been described by Hristov et al. (2005). The present paper describes progress in implementing the model for the macro-region. Matching of the interfacial profiles of the sub-models has not yet been attempted. Instead, a constant macroscopic contact angle is assumed and the contribution of the micro-region to the evaporative heat flux driving bubble growth is estimated by an approximate model. The level-set model for the macro-region has been implemented using COMSOL Multiphysics version 3.2a software. This requires some continuing development of the software and improvements in the graphical presentation of the results, as noted in the paper.

\section{Theory}

A vapour bubble nucleates on a superheated wall with very high thermal conductivity, so that its temperature is approximately constant. Initially the bubble has the shape of a truncated sphere with a defined contact angle, Figure1. The model couples the momentum and continuity equations inside and outside the bubble. The temperature field is modelled outside the bubble only, so at the interface and inside the bubble the temperature is uniform at the saturation temperature. The model takes into account the electric field distortion in the overall domain, together with changes in the electric properties of the liquid with temperature, which generate electric charges in the liquid. Refrigerant R123 at saturation temperature is used as a working fluid because it has a higher electrical conductivity than most
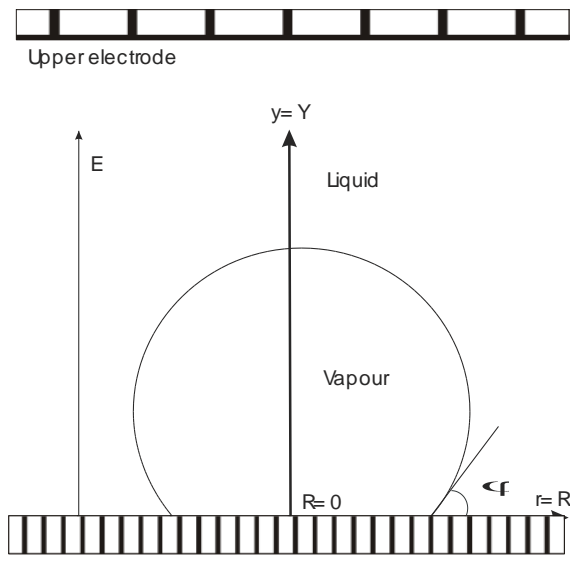
Ground Eectrode dielectric fluids and thus accentuates the effects of charge generation. 
The bubble interface is assumed to have a finite thickness represented by $\phi$-level set function, which is defined as a positive or negative distance from the interface, and an associated Heaviside function $H$ that has value zero in the bulk phases. The negative sign for $\phi$ is chosen for the vapour phase and the positive- for the liquid phase. For the momentum equation for the vapour-interface-liquid region a "one-fluid" formulation has been used:

$\rho\left(\frac{\partial \mathbf{u}}{\partial t}+\mathbf{u} \cdot \nabla \mathbf{u}\right)=-\nabla p+\nabla \cdot \mu \nabla \mathbf{u}+\nabla \cdot \mu \nabla \mathbf{u}^{T}+\rho \mathbf{g}-\rho \beta_{T} \boldsymbol{\Gamma}-T_{s a t} \mathbf{g}-\sigma \kappa \nabla H+\mathbf{F}_{E}$

The curvature at the interface is defined as $\kappa \boldsymbol{\varnothing}=\nabla \cdot\left(\frac{\nabla \phi}{|\nabla \phi|}\right)$

and the other properties as:

$$
\begin{aligned}
& \rho=\rho_{v}+\boldsymbol{\zeta}_{l}-\rho_{v} \vec{H} \\
& \mu=\mu_{v}+\boldsymbol{\iota}_{l}-\mu_{v} \vec{H}
\end{aligned}
$$

where $H$ the Heaviside function is

$$
H=\left\{\begin{array}{cc}
1 & \phi \geq 1.5 h \\
0 & \phi \leq-1.5 h \\
0.5+\phi / 3 h+\sin \& \phi / 3 h \geq \pi_{-}^{-} & |\phi| \leq 1.5 h
\end{array}\right.
$$

The level set function $\phi$ advances as

$$
\frac{\partial \phi}{\partial t}+\mathbf{u}_{\mathrm{int}} \cdot \nabla \phi=0
$$

and has to be reinitialized at each time step as:

$$
\frac{\partial \phi}{\partial t}=\frac{\phi_{0}}{\sqrt{\phi_{0}^{2}+h^{2}}}-|\nabla \phi|
$$

where $\phi_{0}$ is steady state solution of (6).

The last term in (1) is the electric body force -Landau and Lifshitz (1960) defined as

$$
\mathbf{F}_{E}=\rho_{e} \mathbf{E}-\frac{1}{2} E^{2} \nabla \varepsilon+\frac{1}{2} \nabla\left(E^{2} \rho \frac{\partial \varepsilon}{\partial \rho}\right)
$$

where the first term on the right hand side is the electrophoretic force acting on free charges. The second term is due to changes in the electric permittivity of the medium. The third term includes the dielectrophoresis and electrostriction force -Pohl (1978).

The Coulomb force is:

$$
\mathbf{F}_{E}^{C}=\rho_{e} \mathbf{E}
$$

where $\rho_{e}, \mathbf{E}$ are respectively free charge density and the electric field in the domain, which are obtained solving a system of Poisson's equation and the conservation of the electric current $\mathbf{J}$ :

$$
\nabla \cdot \nabla \Phi)-\rho_{e}
$$

$\mathbf{E}=-\nabla \Phi$

$\frac{\partial \rho_{e}}{\partial t}+\nabla . \mathbf{J}=0$

$\mathbf{J}=\rho_{e} \mathbf{u}+\sigma_{\mathbf{e}} \mathbf{E}-$ Ohm law

$\Phi$ is the electric potential and $\varepsilon=\varepsilon_{v}+\epsilon_{l}-\varepsilon_{v} \not H$ is the electric permittivity defined it terms of the level set function properties.

Neglecting the movement of the free charges in steady state Eq. (11) becomes 
$\nabla . \mathbf{J}=\nabla \cdot \mathbf{G}_{\mathrm{e}} \mathbf{E}=\mathbf{E} \cdot \nabla \sigma_{\mathrm{e}}+\sigma_{\mathrm{e}} \nabla \cdot \mathbf{E}=\mathbf{0}$

Combining (9), (10) and (11) gives

$\rho_{e}=\frac{\varepsilon}{\sigma_{\mathrm{e}}} \nabla \Phi . \nabla \sigma_{\mathrm{e}}$

where $\sigma_{e}, \varepsilon$ are assumed to change with the temperature only. Substituting (14) in (9) gives

$\nabla^{2} \Phi+\frac{\nabla \sigma_{\mathrm{e}}}{\sigma_{\mathrm{e}}} \cdot \nabla \Phi=0$

The change of the electric conductivity with the temperature following Turnbull (1968) is:

$\sigma_{\mathrm{e}}=\sigma_{\mathrm{e}, \mathrm{sat}}\left(+\alpha_{K}\left(-T_{\text {sat }}\right)\right.$

where $\sigma_{\mathrm{e}, \text { sat }}$ is the electric conductivity at saturation temperature and $\alpha_{K}$ is a coefficient which has been obtained by $\mathrm{Xu}$ (1995) for R123 refrigerant to be $0.0263 \mathrm{~K}^{-1}$.

The mass conservation equation including the effect of volume expansion due to liquid-vapour phase change is derived from the continuity equation and energy balance across the liquid -vapour interface (Son et al. (1999)):

$\mathbf{m}=\rho \mathbf{a}_{\text {int }}-\mathbf{u}_{-}=\frac{\mathrm{k}_{1} \nabla \mathrm{T}}{\mathrm{h}_{\mathrm{fg}}}$

$\nabla \cdot \mathbf{u}=-\frac{1}{\rho}\left(\frac{\partial \rho}{\partial t}+\mathbf{u} \cdot \nabla \rho\right)=\frac{\mathbf{m} \cdot \nabla \rho}{\rho^{2}}$

where $\mathbf{m}$ is the mass flux of the liquid evaporating at the interface. The vapour produced as a result of evaporation from the microlayer underneath the bubble is added to the vapour volume through the cells adjacent to the heated wall. The evaporation is integrated and expressed as:

$\left(\frac{1}{V_{c}} \frac{d V}{d t}\right)_{m i c}=\frac{\dot{m}_{m i c}}{V_{c} \rho_{v}} \delta_{\varepsilon} \boldsymbol{\phi}_{-}^{-}$

where $\dot{m}_{m i c}=\int_{R_{0}}^{R_{1}} \frac{k_{l} \boldsymbol{\sigma}_{w}-T_{\mathrm{int}}}{h_{f g} \delta} \tau d r$ is the evaporation rate from the microlayer

$V_{c}$ is the volume of the control volume of size $3 h \times h$, where $h$ is the computational grid size, and

$\delta_{\varepsilon} \bar{\phi} \frac{\partial H}{\partial \phi}$ is a smoothed delta function

For these simulations, a spatial average value for the microlayer thickness $\delta=0.8 \sqrt{t \frac{\mu_{l}}{\rho_{l}}}$ derived by Cooper and Lloyd (1969) has been used to calculate integral (20).

The temperature field distribution equations in the domain are:

$\rho c_{P}\left(\frac{\partial T}{\partial t}+\mathbf{u} . \nabla T\right)=\nabla \cdot k \nabla T+\sigma_{e} \mathbf{E}^{2}$ for $\phi>0$ (in the liquid outside the bubble)

$T=T_{s a t} \boldsymbol{Q}_{v}$

for $\phi \leq 0$ (inside the bubble and at the interface)

$k=k_{l} H^{-1}$

The term added to the right-hand side of (22) represents Joule heating in the liquid and can be neglected in the case of R123 following Allen and Karayiannis (1995).

The boundary and initial conditions are described below, see Figure 1.

$\begin{array}{lll}u=v=0, T=T_{w}, \frac{\partial \phi}{\partial y}=-\cos \varphi, \Phi=0 & \text { at } & y=0 \\ \frac{\partial u}{\partial y}=\frac{\partial v}{\partial y}=\frac{\partial \phi}{\partial y}=0, T=T_{\text {sat }}, \Phi=\Phi_{0} & \text { at } & y=Y\end{array}$ 
$u=\frac{\partial v}{\partial r}=\frac{\partial T}{\partial r}=\frac{\partial \phi}{\partial r}=\frac{\partial \Phi}{\partial r}=0 \quad$ at $\quad r=0, R$

where $\varphi$ is the apparent contact angle (Figure 1), for which a value is chosen based on experimental observations and is kept constant. For the final model in which the macro- and microregions will be matched, the value of the apparent contact angle will change in time. The other boundary conditions refer to the axisymmetry of the problem.

Initial conditions: $u(t=0)=0 \quad v(t=0)=0 \quad T(t=0)=T_{\text {sat }} \Phi(t=0)=0 \quad \phi(t=0)=\psi \varangle, y_{\text {_( }}(28)$

where $\psi$ is the initial shape of the bubble, usually a truncated sphere with a defined radius.

The temperature field at $t=0$ for the sample calculations in this paper is defined by Holman (1986),

$$
\frac{T-T_{w}}{T_{s a t}-T_{w}}=\frac{3}{2} \frac{y}{\delta_{T}}-\frac{1}{2}\left(\frac{y}{\delta_{T}}\right)^{3}
$$

where $\delta_{T}$ is thermal boundary layer thickness, evaluated using the correlation for the turbulent natural convection on a horizontal plate (Kays and Crawford (1980)) as

$\left.\delta_{T}=7.14\left(\frac{v_{l} \alpha_{l}}{g \beta_{T} \boldsymbol{\zeta}_{w}-T_{\text {sat }}}\right)\right)^{\frac{1}{3}}$

The calculation of the temperature-dependent changes in $\nabla \sigma_{\mathrm{e}}$ in (14) from the instantaneous temperature field calculated from (22) has not yet been fully implemented because it requires significant rewriting of the CFD code. An approximate implementation has been adopted for the particular conditions of the example in this paper. When the initial radius of the bubble is taken as 8 times the initial thickness of this boundary layer, as in Figure 2, it is found that there is negligible change in the temperature field calculated by (22), so that combination of (9), (15), (16) and (29) gives an analytical expression for the charge density that is independent of time and which can be used directly in the unmodified CFD code:

$$
\rho_{e}=\frac{\frac{3}{2} \frac{\varepsilon}{\delta_{T}}\left(1-\left(\frac{y}{\delta_{T}}\right)^{2}\right) \frac{\partial \Phi}{\partial y}}{1-\frac{3}{2} \frac{y}{\delta_{T}}+\frac{1}{2}\left(\frac{y}{\delta_{T}}\right)^{3}+\frac{1}{\alpha_{K} \boldsymbol{\sigma}_{w}-T_{\text {sat }}}-}
$$

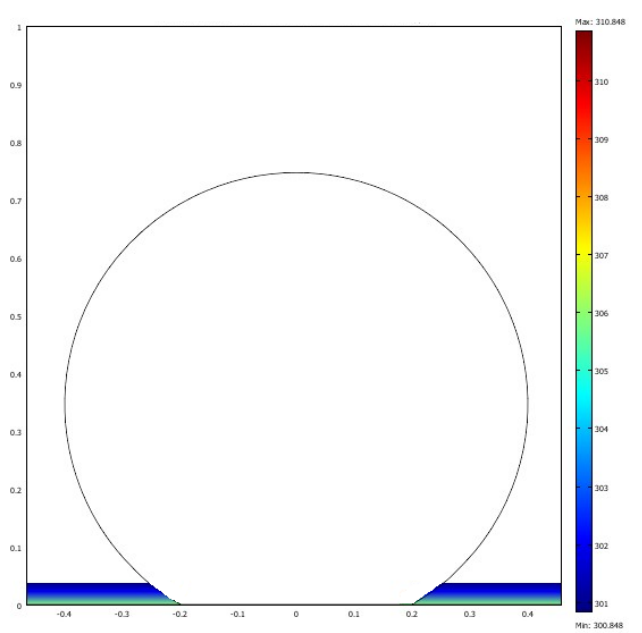

Figure 2. Initial thickness of the thermal boundary layer

\section{Results and discussion}

The effect of the electric field on the shape and stability of a bubble has been analysed first by Cheng and Chaddock (1984) and more recently by Herman and Iacona (2004). They both used the same idea that the equilibrium shape of the bubble corresponds to the extremum of the thermodynamic potential (the Gibbs free energy for a vapour in a liquid). They did not consider the electrohydrodynamics of the problem. The following results are obtained through carrying out numerical simulations considering the full electrohydrodynamics of the problem. The properties of saturated refrigerant R123 at $1 \mathrm{~atm}$ are used, with a contact angle $\varphi=30$ deg and a wall superheat

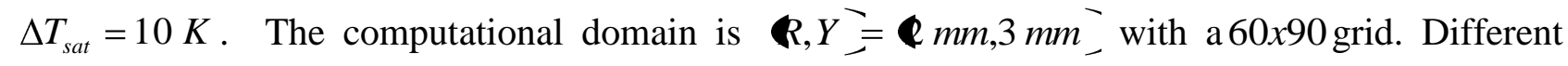
electric field strengths up to $10 \mathrm{MV} / \mathrm{m}$ have been applied in order to investigate the effect of the electric field strength on bubble growth and detachment. The initial bubble radius is set at $0.4 \mathrm{~mm}$, much larger than the typical radius of a nucleus. This is compatible with the coarse computational 
grid and satisfies the condition for specification of the charge density by the simplified expression in (31). Simulation of a full bubble cycle from nucleation to departure will eventually require grid refinement for the early stages. The examples in this paper focus on the effect of the electric field on bubble departure.

In Figure 3 bubble growth and detachment in time for $t=3,4,5 \mathrm{~ms}$ (up to bottom) for different applied electric fields ( $E=0,5,10 \mathrm{MV} / \mathrm{m}$ from left to right) is presented. In all the simulations we start from one and the same initial bubble radius of $0.4 \mathrm{~mm}$. The results show that the elongation of

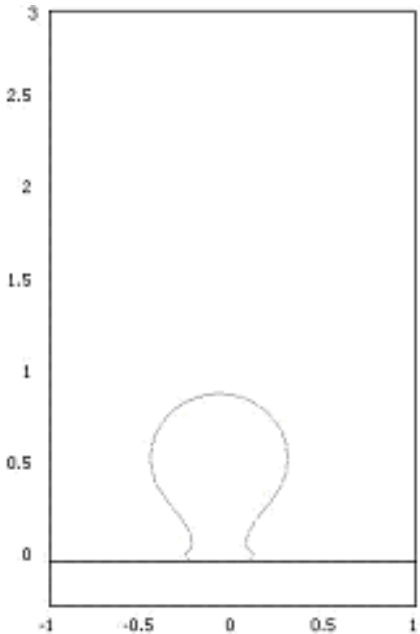

(a)

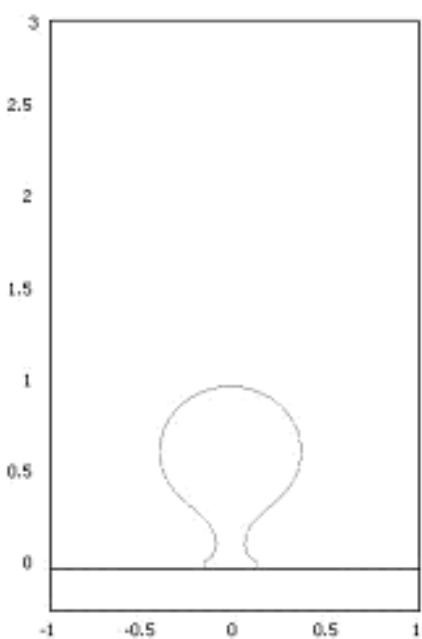

(b)

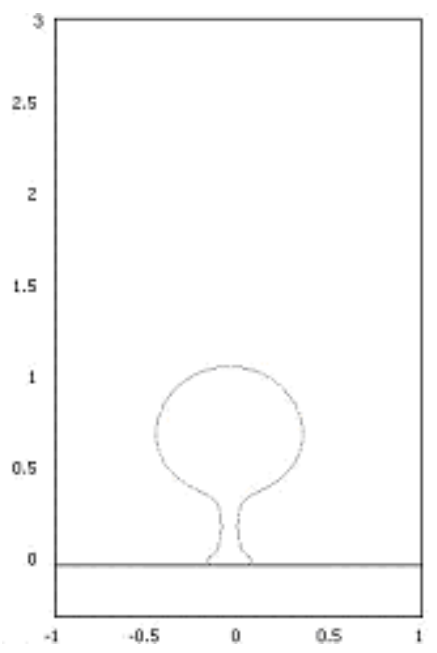

(c)

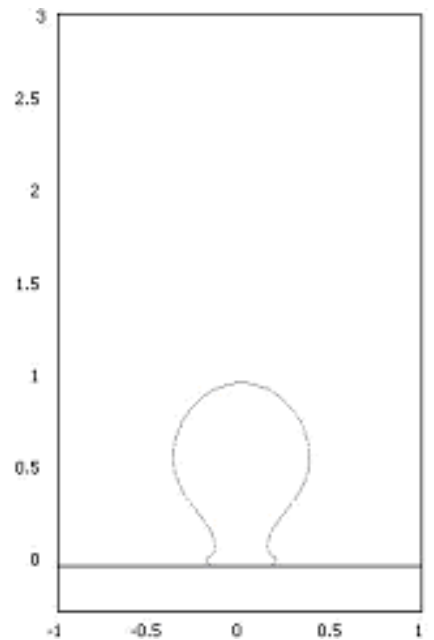

(d)

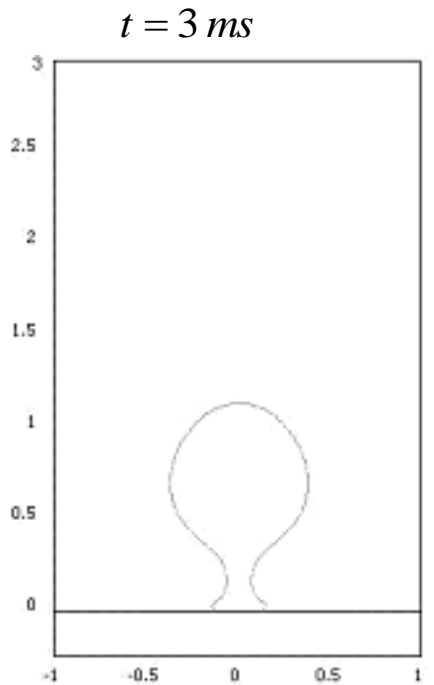

(e)

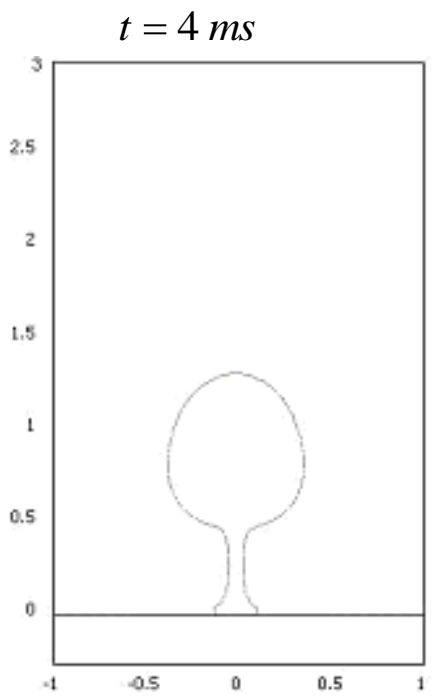

(f)

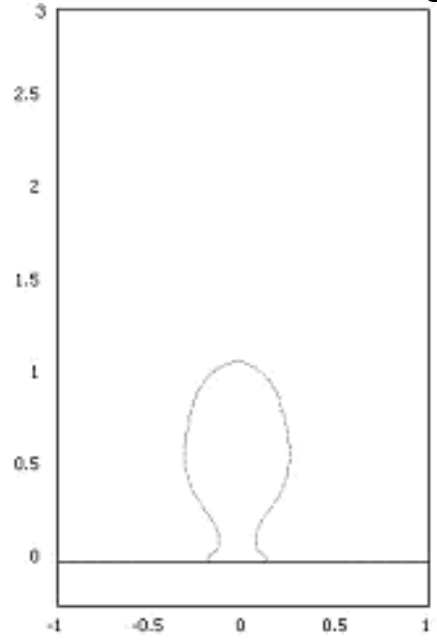

(g)

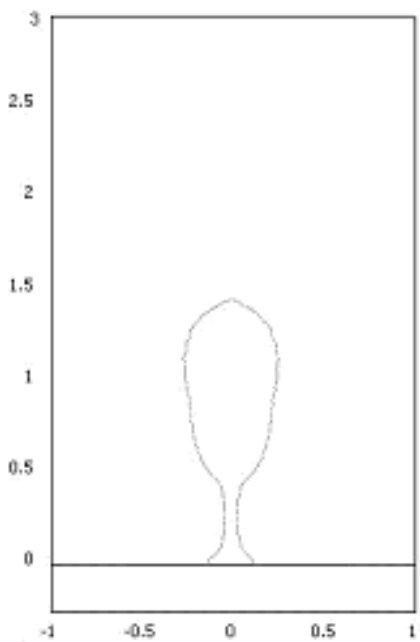

(h)

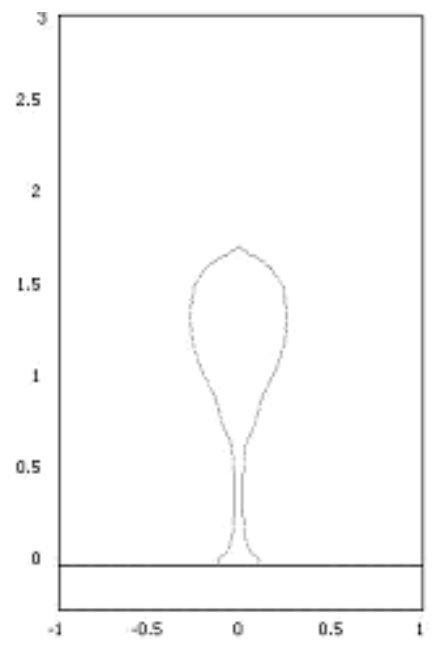

(i) 


$$
t=5 \mathrm{~ms}
$$

Figure 3. Bubble growth for $E=0 M V / m-(a, b, c) ; 5 M V / m-(d, e, f) ; 10 M V / m-(g, h, i)$

electric field strength applied in $t=3,4,5 \mathrm{~ms}$ (up to bottom).

The bubble in the direction of the electric field is more pronounced with stronger electric fields. Although this trend has been demonstrated previously, an important difference between the presented results and previous works is that in this investigation considers the complete electrohydrodynamics conditions and the temperature field as well as heat transfer of the process, which makes the model closer to the real situation of boiling heat transfer. The results revealed a threshold for the electric field strength of $3 \mathrm{MV} / \mathrm{m}$ below which the effect of the electric field on the deformation of the bubble is negligible.

During the final stages of bubble growing just before detachment, the dominance of buoyancy over the surface tension accelerates as the bubble base shrinks. A small neck develops, remains for very short time and breaks off due to the strong imbalance of the forces. Unlike the model of Son et al. (1999), in which the authors imposed an artificial condition for minimum bubble base size to force detachment, in the present model no such condition was necessary.

In Figure 4, the bubble shape immediately after detachment is shown. The bubble detachment time as well as the shape of the detached bubble change due to electric field effect. In all the cases it is found that a small amount of vapour remains on the heated surface, which starts to grow and closes the cycle of bubble formation and detachment in nucleate boiling. More realistic conditions for the initial radius of the bubble and the temperature field for the next bubble growing from the residual vapour will be developed in the next stage of the work.
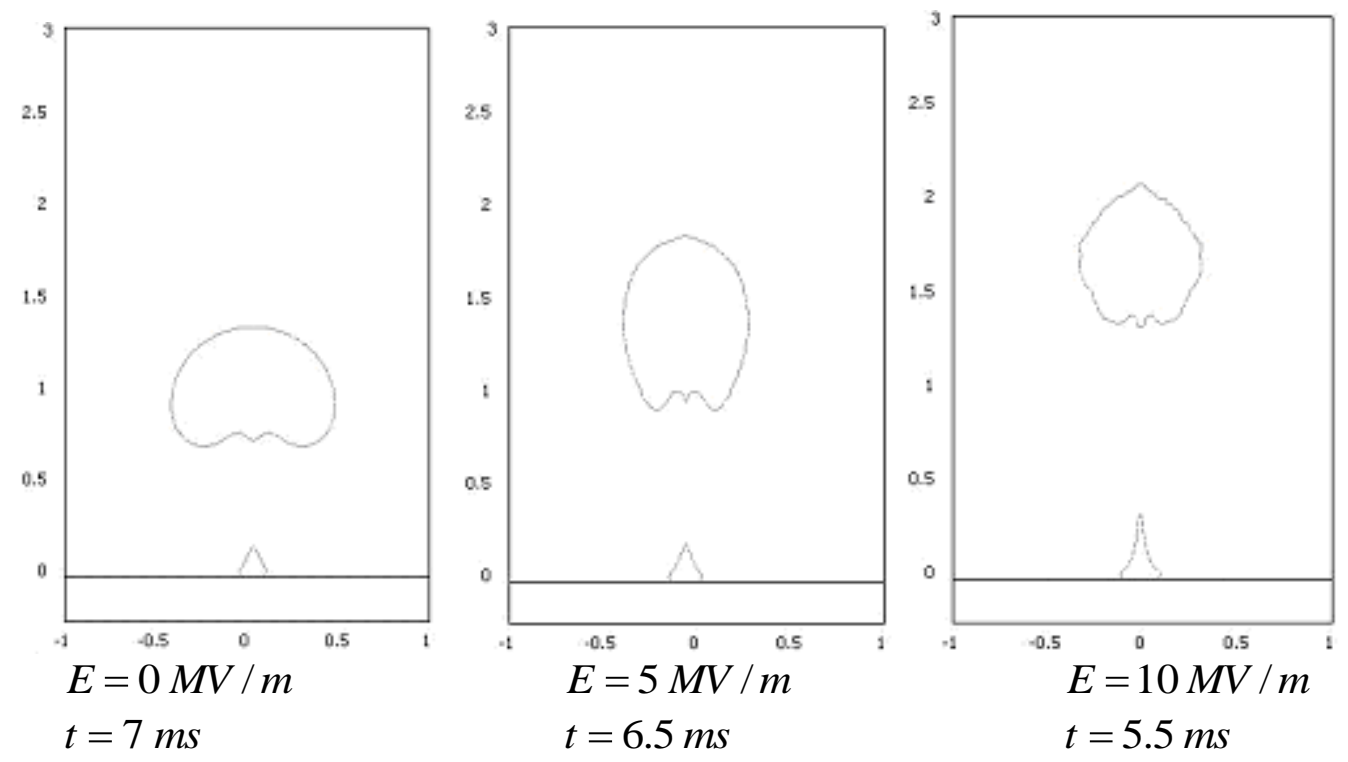

Figure 4. Bubble shape immediately after detachment for $E=0,5,10 \mathrm{MV} / \mathrm{m}$ electric field strength applied.

In Figure 5, the detachment time and the volume ratio between detachment volume without and with an electric field are plotted as functions of electric field strength. The values of bubble volume are at present subject to numerical errors in the conservation of mass as the bubble deforms, a characteristic of the level-set method. Corrections for this are being developed. When an electric field of $10 \mathrm{MV} / \mathrm{m}$ is applied, the bubble detaches in a shorter time $(t=5.2 \mathrm{~ms})$ and at a smaller volume, than with $5 \mathrm{MV} / \mathrm{m}(t=6.1 \mathrm{~ms})$, or without electric field $(t=6.7 \mathrm{~ms})$. This effect has been observed by many authors experimentally and for R123 recently by Zhao et al. (2006). The result of a higher bubble detachment frequency is likely to be an enhancement of the heat transfer. In order to quantify the effect of the electric field on the deformation of the bubble, an aspect ratio 
$\frac{a}{b}$ between the major and the minor axis of the spheroid as introduced by Cheng and Chaddock (1984) has been calculated, shown in Figure 6 for $E=0,5,10 \mathrm{MV} / \mathrm{m}$.

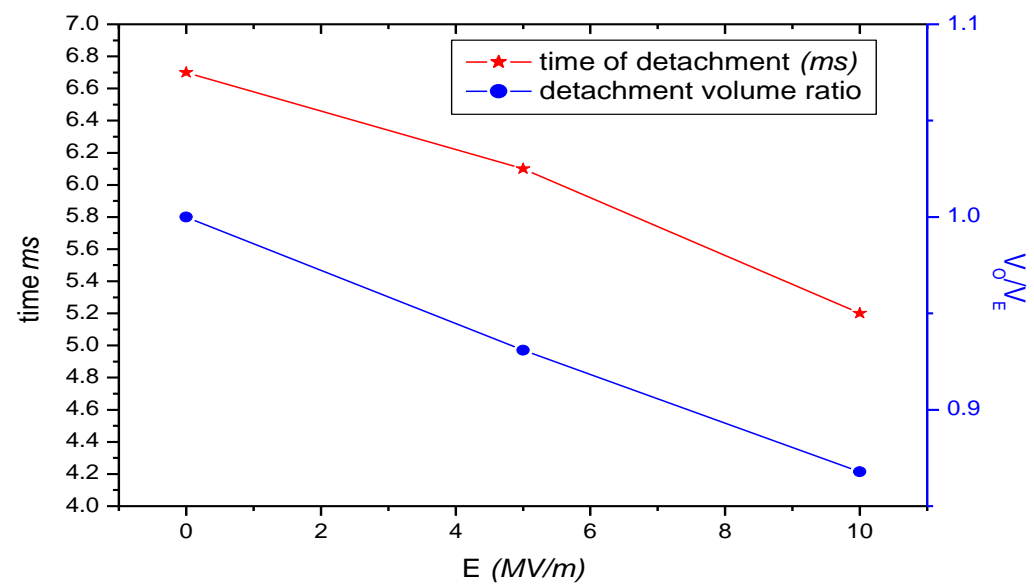

Figure 5. Change of the bubble detachment time and bubble detachment volume ratio with the electric field strength.

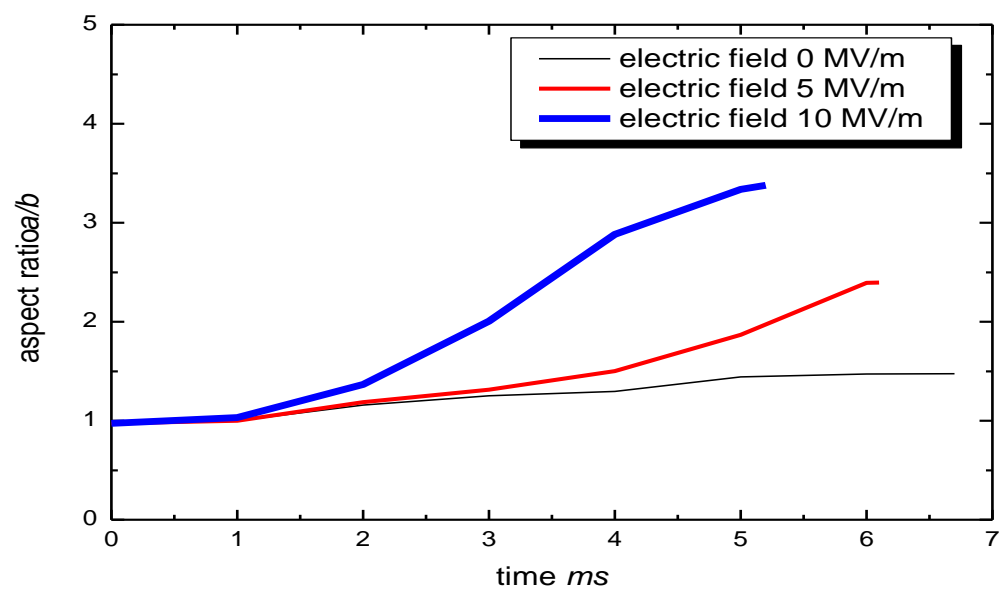

Figure 6. Aspect ratio in time with $E=0,5,10 \mathrm{MV} / \mathrm{m}$ electric field strength.

The results for the aspect ratio in Figure 6 show that the electric field strength is the main factor causing bubble elongation in the direction of the electric field. The end points of the curves represent the instant at which the bubble detaches.

Figure 7 presents the electric field strength iso-levels for different electric fields applied. In case of isothermal and free of bubbles liquid, the electric field distribution will be identical throughout the domain. On Figure 7 there is distortion in the vicinity of the bubble and the maximum gradient of the electric field occurs at the top of the bubble. Electric field distribution outside as well as inside the bubble is due to the implementation of the idea of Karayiannis and Xu (1998), that there is no need to apply boundary condition of type $\frac{\partial \Phi}{\partial n}=0$ at the interface of the bubble. That condition differs from most of the previous works done on the effect of the electric field on bubble deformation, because they predetermine the solution to be in tangential direction and the electric field inside the bubble to be zero. Another novelty in this work worth mentioning is the "one-fluid" formulation of the electric problem, which allows it to be solved with level set method. The idea of the formulation is that electric permittivity changes smoothly from $\varepsilon_{v}$ to $\varepsilon_{l}$ at the interface as 
described in paragraph 2. That also gives an easy mathematical implementation of the second term in (7):

$\left.-\frac{1}{2} E^{2} \nabla \varepsilon=--\frac{1}{2} E^{2} \nabla \epsilon_{v}+\epsilon_{l}-\varepsilon_{v} \not H\right)-\frac{1}{2} E^{2} \epsilon_{l}-\varepsilon_{v} \delta_{\varepsilon} \mathbf{Q} \nabla \varphi$

(32) together with the Clausius -Mossotti relationship $\left(\frac{\varepsilon_{l}}{\varepsilon_{v}}-1\right)\left(\frac{\varepsilon_{l}}{\varepsilon_{v}}+2\right)^{-1}=3\left(\frac{\partial \varepsilon}{\partial \rho}\right)_{T} \rho$

give $\frac{1}{2} \nabla\left(E^{2} \rho\left(\frac{\partial \varepsilon}{\partial \rho}\right)_{T}\right)=\frac{\varepsilon_{0}}{6} E^{2}\left(\frac{\varepsilon_{l}}{\varepsilon_{v}}-1\right)\left(\frac{\varepsilon_{l}}{\varepsilon_{v}}+2\right)$

for the third term in (7) (see Karayiannis and Xu (1998)) enable the model to take into account all the terms into electric body force (7) and makes the modelling more realistic. The above approach results in smooth distortion of the electric field passing through the interface of the bubble as shown on Figure 7 for different electric field strengths.
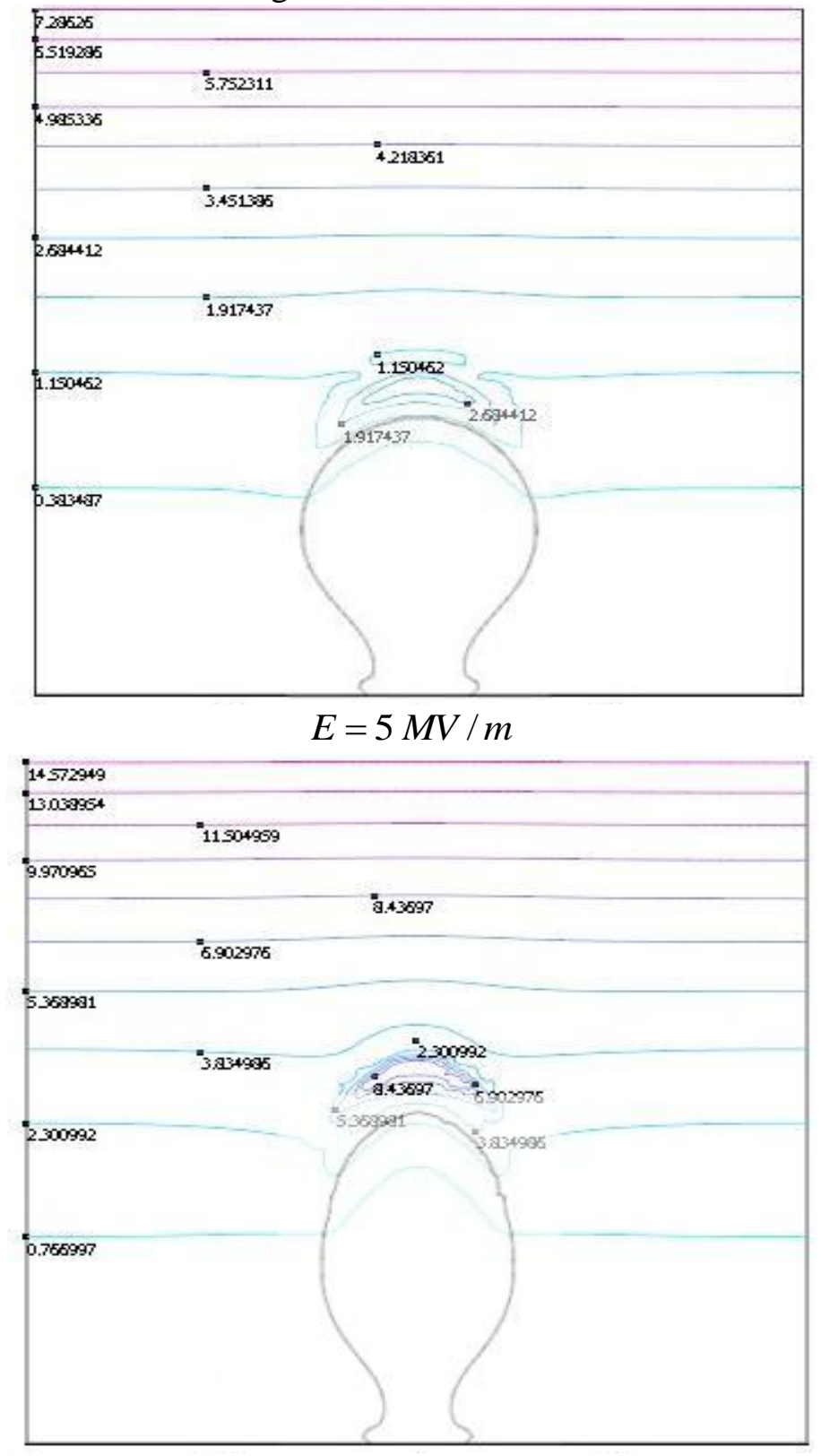

$E=10 \mathrm{MV} / \mathrm{m}$

Figure 7. Electric field distortion at $t=3 \mathrm{~ms}$. 
Figure 8 shows the velocity field around the bubble during its growth. These results show higher velocities for higher electric fields. This implies an enhancement of the convective heat flux and overall heat transfer improvement induced by the electric field.

Figure 9 shows wall heat flux distribution prior to bubble detachment at different electric field strengths. The results demonstrate low sensitivity of the wall heat flux to the strength of the electric field during this part of the bubble cycle. The increase in the experimental heat transfer coefficient for R123 at similar electric field strengths observed by Zhao et al. (2006) may due either to effects during the initation of bubble growth or increases in bubble frequency.
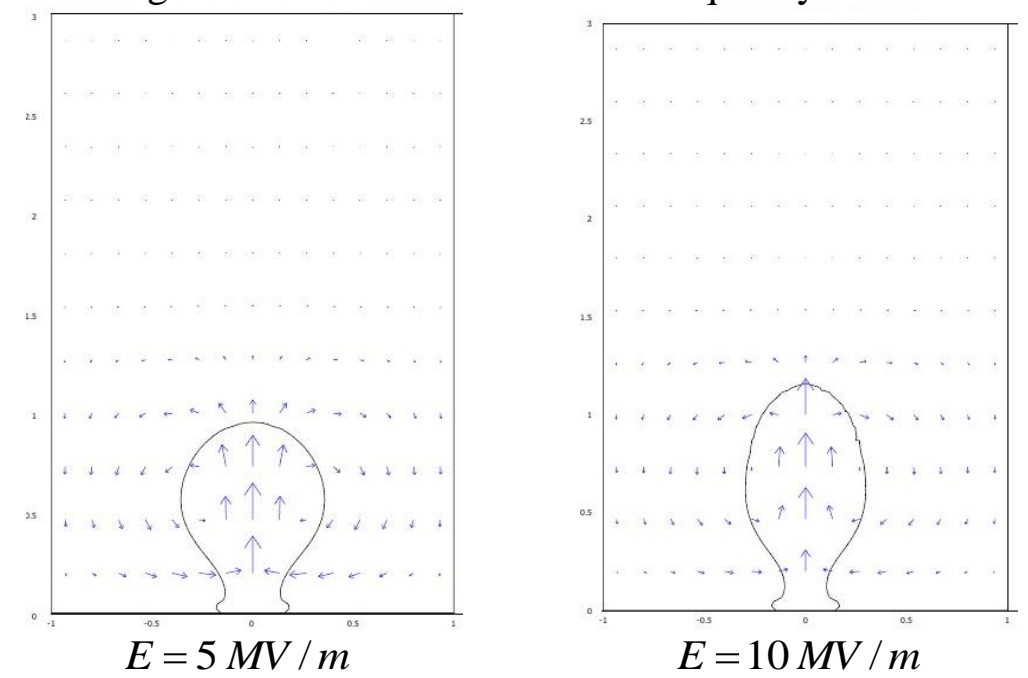

Figure 8. Velocity field distribution for $E=5,10 \mathrm{MV} / \mathrm{m}$ at $t=3 \mathrm{~ms}$.
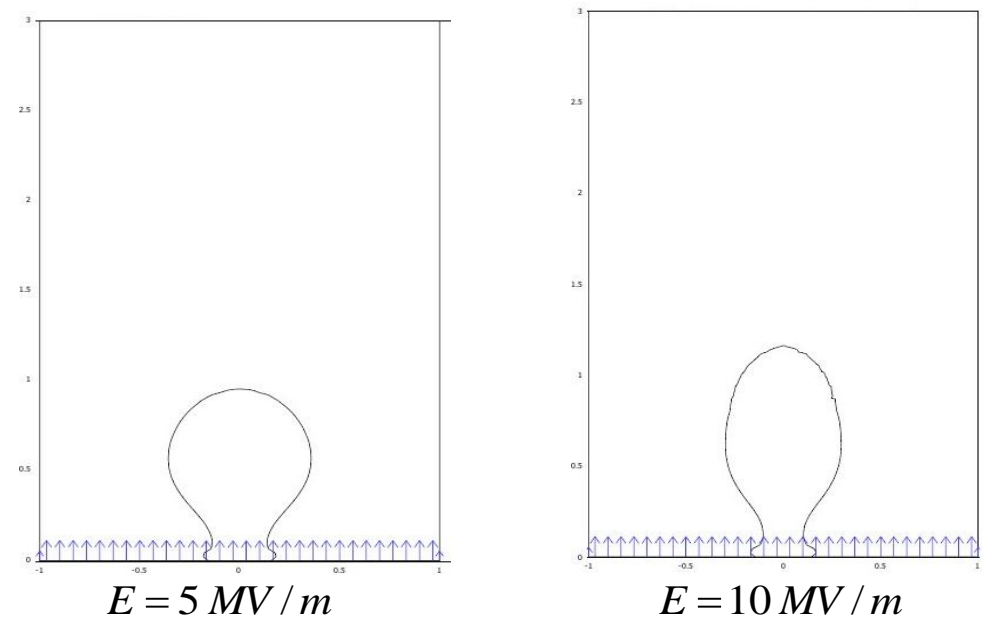

Figure 9. Wall heat flux distribution for $E=5,10 \mathrm{MV} / \mathrm{m}$ at $t=3 \mathrm{~ms}$.

\section{Conclusions}

A macro-region model describing the deformation, growth and detachment of a vapour bubble in the nucleate boiling regime with an applied electric field has been developed and solved using the level set method. It describes the full electrohydrodynamics of the process including free charge generation, as well as the temperature field distribution in the domain. The proper prediction of the electric field distribution is a fundamental problem in EHD research; any simplification can affect the accuracy of the results. The presented model considers all the terms in electric body force and is not restricted to small deviations from the spherical shape of the bubble. Electric field distribution inside the bubble is also included. The elongation of the bubble in the direction of the electric field, the increase of the aspect ratio as well as stronger convective heat flux with strong electric field are amongst the results in good agreement with the experimental observations. The results presented are 
a solid foundation for further development of the EHD model to include coupling of the macrolayer region to a micro-region model.

Acknowledgement

This work was supported by the Engineering and Physical Sciences Research Council, Grant GR/S411821/01.

\section{REFERENCES}

Allen, P.H.G., Karayiannis T. G., 1995, Electrohydrodynamic enhancement of heat transfer and fluid flow-Review paper, Heat Recovery Systems \& CHP, vol. 15, No. 5, 389-423.

Cheng, K.J., Chaddock, J.B., 1984, Deformation and stability of drops and bubbles in an electric field, Phys. Lett. 106 A, 51-53.

Cooper, M.G., Lloyd, A.J.P., 1969, The microlayer in nucleate pool boiling, Int. J. Heat Mass Transfer, Vol. 12, 895-913.

Di Marco, P., Grassi W., 1999, EHD Effects on pool boiling in reduced gravity, Proceedings of the 5th ASME/JSME Joint Thermal Engineering Conference, March 15-19, 1999, San Diego, California.

Herman, C., Iacona, E., Modelling of bubble detachment in reduced gravity under the influence of electric fields and experimental verification, Heat and Mass Transfer 40 (2004), 943-957.

Holman, J.P., Heat transfer, McGraw-Hill (1986).

Hristov, Y., Zhao, D., Sefiane, K., Karayiannis, T.G., Kenning, D.B.R., Critical heat flux (CHF) modelling in pool boiling heat transfer, including the effect of an electric field, Proceedings of 9th UK National Conference of Heat Transfer, Manchester (2005).

Karayiannis, T.G. (1998) EHD boiling heat transfer enhancement of R123 and R11 on a tube bundle, Applied Thermal Engineering 18, 809 - 817.

Karayiannis, T.G., Allen P.H.G., Electro-hydrodynamic enhancement of two-phase heat transfer, Eurotech Direct Cong Thermofluid Eng (1991), 165-181.

Karayiannis, T.G., Xu, Y., Electric field effect in boiling heat transfer. Part A: Simulation of the electric field and electric forces, Enhanced Heat Transfer 5 (1998), 217-229.

Kays, W.M., Crawford, M.E., Convective heat and mass transfer, McGraw-Hill (1980), p. 328.

Landau, L.D., Lifshitz, E.M., Electrodynamics of continuous media, Addison-Wesley, Reading, Masschussetts (1960).

Laohalertdecha, S., Naphon, P., Wongwises, S., A review of electrohydrodynamic enhancement of heat transfer, Renewable and Sustainable Energy Reviews (2005), In Press, Corrected Proof.

Ogata J., Yabe A., Basic study one the enhancement of nucleate boiling heat transfer by applying electric fields, Int. J. Heat Mass Transfer 36 (1993), 742-745.

Oh, S.D., Kwak, H.Y., A study of bubble behaviour and boiling heat transfer enhancement under electric field, Heat Transfer Engineering 21 (2000), 33-45.

Pohl, H. A. Dielectrophoresis: The Behaviour of Neutral Matter in Non-uniform Electric Fields, Cambridge University Press, (1978).

Son, G., Dhir, V.K., Ramanujapu, N., Dynamics and heat transfer associated with a single bubble during nucleate boiling on a horizontal surface, J Heat Transfer 121 (1999), 623-631.

Turnbull, R.J., Electroconvective instability with a stabilizing temperature gradient. I. Theory, Physics of Fluids vol. 11, number 12 (1968), 2588-2596.

Verplaetsen, F.M., Berghmans, J.A., Film boiling of an electrically insulating fluid in the presence of an electric field, Heat and Mass Transfer 35 (1999), 235-241.

$\mathrm{Xu}, \mathrm{Y}$., Effects of electric fields on pool boiling heat transfer, Ph. D. Thesis, South Bank University, UK (1995).

Zaghdoudi, M.C., Lallemand, M., Study of the behaviour of a bubble in an electric field: steady shape and local fluid motion, Int. J. Therm. Sci. 39 (2000), 39-52. 
Zaghdoudi, M.C., Lallemand, M., Electric field effects on pool boiling, Journal of Enhanced Heat Transfer 9 (2002), 187-208.

Zhao, D., Karayiannis, T.G., Kenning, D.B.R., Sefiane, K., Hristov, Y. (2005) 6th International Conference on Boiling Heat Transfer. May 7-12, 2006 Spoleto, Italy

Zhang, H.B., Yan, Y.Y., Hull, J.B., Numerical simulations of EHD, effects on a single bubble attached on a horizontal surface in nucleate boiling, Proceedings of 8-th UK National Conference of Heat Transfer, Oxford (2003). 